\title{
Gene research products
} Tools for genetic research covered in this feature include a hand-held laboratory 'assistant', high-grade nucleotides
for PCR, a probe for murine telomeres, a catalogue for antisense oligonucleotides and new gel loading pipette tips.

\section{CEQ 2000}

From Beckman Coulter

Capillary electrophoresis DNA sequencing in a 96-well format

Utilizing capillary electrophoresis (CE) technology with laser-induced fluorescence detection, this system features four-colour, dideoxy-terminator chemistry and operates in a microplate format. CEQ handles more of the activities of dideoxy-terminator sequencing, from pre-run setup, sample denaturation, gel replenishment, injection and separation, to final determination of base sequence, with little or no human intervention. Using four dyes per capillary to help deliver fast turnaround and high throughput, a final report calling over 500 bases on eight samples can be produced in just $2 \mathrm{~h}$, and on a complete 96-well plate in $24 \mathrm{~h}$. In addition, the system's software measures the accuracy of each base call, eliminating the need to access a separate software program for accuracy determination. CEQ samples have format compatibility with the Biomek 2000 laboratory automation workstation and the Multimek 96 automated 96 channel pipettor. Reader Enquiry No. 100

\section{MBA 2000 system}

From Perkin-Elmer

A new productivity tool for genetic analysis, biotechnology and drug discovery

The molecular biology assistant (MBA) instrument is a compact computational system that consolidates and organizes routine and customized functions used in virtually all protocols that employ biochemicals. The system employs an intuitive, touchscreen display and has no moving parts, all in a nine-inch wide, low-profile package. Choices are made simply with a touch-toselect action and the user is prompted as needed. From estimating reagent usage costs to checking absorbance, from setting up serial dilutions and PCR master mixes to designing new oligonucleotides for PCR or DNA sequencing, the MBA 2000 system is

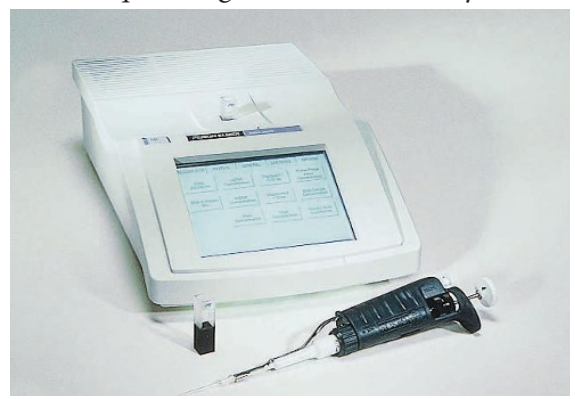

Getting an MBA from Perkin-Elmer may pay off. designed to make computations and quantification accessible and rapid. It can be customized for specific analyses and determinations.

Reader Enquiry No. 101

\section{Platinum PCR products}

From Gibco BRL

New literature covering the Platinum Taq polymerase and its corresponding antibody

These products provide automatic hotstarts for greater specificity in PCR procedures. The polymerase-antibody mixture is said to eliminate the need to add antibody before assembling PCR. Provided in Taq equivalent units, the antibody is supplied as a solution to be added to PCR by the user. There is said to be real-time activation of Platinum Taq DNA polymerase with this system, and the Taq antibody is said to eliminate pre-PCR misprimings. Automatic hotstarts activate Taq at $94{ }^{\circ} \mathrm{C}$ during the first step of PCR denaturation. The product design is said to ensure stable storage by completely inhibiting the enzyme until use. According to the manufacturer, reactions can be initiated at room temperature, and amplify templates up to $5 \mathrm{~kb}$. Reader Enquiry No. 102

\section{PCR-grade nucleotides}

From Boehringer Mannheim

For applications that require high-quality reagents and provide quality results

These deoxynucleotides have been HPLC purified to high standards (HPLC area $\geq 99$ percent, dNDP area $\geq 0.9$ per cent) and are supplied as ready-to-use sodium salt solutions. Each lot is function tested in an RTPCR assay to confirm the absence of RNase, DNase and nicking activity. These reagents should prove useful in reverse transcription (RT), PCR, RT-PCR and cycle sequencing. The nucleotides (dATP, dCTP, dGTP, dTTP and dUTP) can be purchased in $25-\mu \mathrm{mol}$ and $1,250-\mu \mathrm{mol}$ sizes.

Reader Enquiry No. 103

\section{T3 thermocycler}

From Biometra

Part of a new generation of multiblock instruments

These instruments feature three independently operating sample blocks within one compact housing. Each block is equipped with it own pressure-optimized, heated lid. This can be set to run its own individual programme, allowing the user to run three different protocols at any one time. The

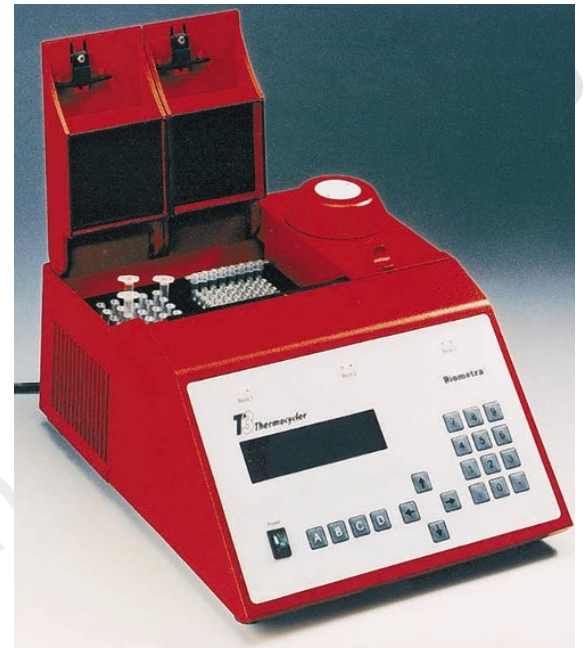

Biometra's T3 three-in-one thermal cycler.

triple format can also significantly increase the capacity of a laboratory by enabling three users to work simultaneously on one instrument. The system also has the ability to optimize run conditions quickly .

Reader Enquiry No. 104

\section{Blotting materials}

\section{Hybond-XL}

From Amersham Pharmacia Biotech A new positively charged nylon membrane for radioactive nucleic acid blotting

This is the company's first membrane designed specifically for radioactive nucleic acid blotting. It is built to provide an improved signal-to-noise ratio in applications such as Southern blots, northern blots and colony/plaque lifts. This means that shorter exposure times can be used and that more information can be obtained. It is also suitable for re-probing. Moreover, improvements in signal can be obtained if protocols are optimized for the new membrane, which is said to be well suited for use with such products as Rediprime, Megaprime and ${ }^{32} \mathrm{P}$ for Southern blotting applications. Hybond-XL is quality controlled to ensure detection of $0.1 \mathrm{pg}$ of genomic DNA in a Southern blot with a four-hour exposure. Reader Enquiry No. 105

\section{Protran BA 83 and 85}

From Schleicher \& Schuell

Nitrocellulose transfer membranes for blotting techniques in life-science applications Protran BA $83(0.2 \mu \mathrm{m})$ and BA 85 $(0.45 \mu \mathrm{m})$ are said to be versatile membranes that are well suited to Southern, northern and western blotting, diagnostic antigen 
detection and colony/plaque lifts. The membrane should have excellent handling properties as a result of the very high mechanical stability of the nitrocellulose material. It has an increased tensile strength, stated to be double that of previous membranes, together with low background noise. The high mechanical stability of this pure nitrocellulose membrane gives improved handling characteristics with reduced brittleness.

Reader Enquiry No. 106

\section{AnRiNA world}

\section{RNApure}

From GenHunter

For the extraction of total RNA from tissues or cells

GenHunter has released its new RNApure reagent - a simple monophasic solution for rapid, one-step isolation of intact total RNA. According to the manufacturer, the isolated RNA is of high quality and can be used for differential display, northern or reverse northern-blot analysis. Both 50-ml and $100-\mathrm{ml}$ containers are available, and come with detailed protocol.

Reader Enquiry No. 107

\section{RNA labelling systems \\ From Enzo Diagnostics \\ Newly formatted RNA transcript labelling}

These systems have been formulated and optimized for use with microchip assays, gene expression analyses, RNase protection assays and other non-radioactive applications. RNA probes can be labelled for use in in situ hybridization assays, Southern and northern blot analyses, and other hybridization procedures. Reagents and a detailed protocol are provided with these systems, and each provides a reagent kit containing enzymes (T3/T7, T7/SP6, T3, T7 or SP6 RNA polymerases), buffers and control templates. There is also a choice of nucleotide labelling mix, which includes biotin-11-UTP, biotin-16-UTP, biotin-11CTP, biotin-17-ATP, digoxygenin-11-UTP or fluorescein-12-UTP.

Reader Enquiry No. 108

\section{DWA tools}

Pan centromeric DNA probe

From Oncor

An addition to the company's line of mouse paint and unique sequence DNA probes

Intended for use in fluorescence in situ hybridization (FISH), this new biotinlabelled probe targets the centromeres of all twenty-one mouse chromosomes. According to the manufacturer, it takes just under three hours for mouse bone marrow assayed with the probe to yield a large, bright fluorescent spot or signal at the site of each centromere, with a clean background. The mouse

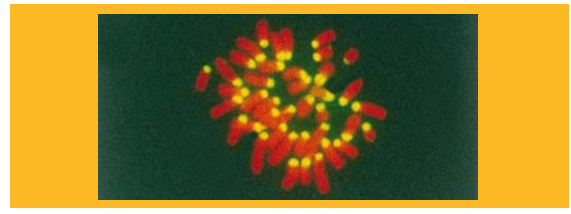

FISHing for a mouse centromere with Oncor .

pan-centromeric DNA probe is biotinlabelled and supplied pre-mixed in hybridization buffer in a quantity of $500 \mu \mathrm{l}$. This amount is sufficient to assay $20-50$ slides, depending on hybridization area. Reader Enquiry No. 109

\section{cDNA libraries}

From Stratagene

The reagents needed for constructing a cDNA library

Stratagene has optimized its cDNA synthesis kit to provide full-length, high-quality cDNA to researchers. Combined with cloning vectors, high-efficiency lambda packaging extracts and specialized plasmid transformation technology, these cDNA kits can provide users with a large, complex cDNA library. Optimization of the synthesis process includes the first-strand synthesis, the directional cloning method and even the polishing reaction that is used to create blunt ends for efficient adapter ligation. Efficient synthesis from mRNA to cDNA is performed using a recombinant Moloney murine leukaemia virus reverse transcriptase (MMLV-RT) employed for its ability to produce large quantities of high-molecular-weight cDNA.

Reader Enquiry No. 110

\section{Biognostik catalogue}

From Chemicon

A range of antisense oligonucleotides capable of selective inhibition of gene expression

Biognostik offers a large selection of antisense oligonucleotides, with brand names such as PCR PrimeR and HybriProbes. These products should prove useful in a wide variety of biological research areas. Custom oligonucleotide kits designed against a specific target gene are also available.

Reader Enquiry No. 111

\section{MPG streptavidin and avidin}

From Quantum Biotechnologies

Magnetic porous glass particles with streptavidin or avidin covalently bound

This system is designed to allow for fast, reliable magnetic separation of biotinylated molecules. The biotin binding capacity of MPG strepavidin and avidin $\left(K_{\mathrm{d}}=10-15 \mathrm{M}\right)$ is retained under a variety of conditions, including treatment with solvents (ethanol and DMSO, for example) and detergents (such as, Triton X-100 and Tween 20). Routine applications include solid-phase
ADVERTISEMENTS

\section{GPIERCE}

SuperSignal ${ }^{\circledR}$ ULTRA

Chemiluminescent Substrate *

Femtogram Sensitivity for HRP Detection in

Western Blotting

Features/Benefits:

- Mid-femtogram-level $\left(10^{-15}\right)$ sensitivity at an economical price

- 24-hour light emission allows for multiple exposures

- Recommended primary antibody dilution is 1:5,000-1:50,000; recommended secondary antibody dilution is $1: 50,000-1: 250,000$

- 24-hour working solution stability

- Allows for stable hard-copy results on film, rapid processing and high resolution

Tel: $800-874-3723$ or $815-968-0747$ - Fax: 815-968-7316 CS@piercenet.com • Internet: www.piercenet.com

* Patent Pending READER ENQUIRY NO.34

\section{LLPOSOMES}

REPRODUCIBLE - HOMOGENEOUS EXTREMELY RAPID

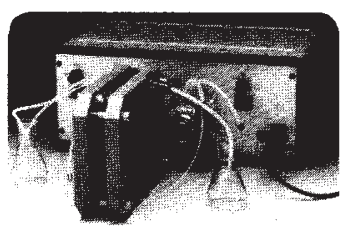

Our new LIPOSOMAT guarantees rapid $(5 \mathrm{ml}$ within 60 minutes) preparation of uniformly sized unilamellar liposomes. Liposome size is selectable between 25 and ca. $600 \mathrm{~nm}$ diameter. Sample volume is normally $5-10 \mathrm{ml}$ or up to $200 \mathrm{ml}$ if used in combination with an additional instrument. Lipid concentration can be up to $300 \mathrm{mg} / \mathrm{ml}$.

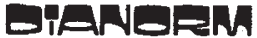

P.O. Box 650126, D-81215 Munich For USA call 1-800 Liposome

READER ENQUIRY NO.62

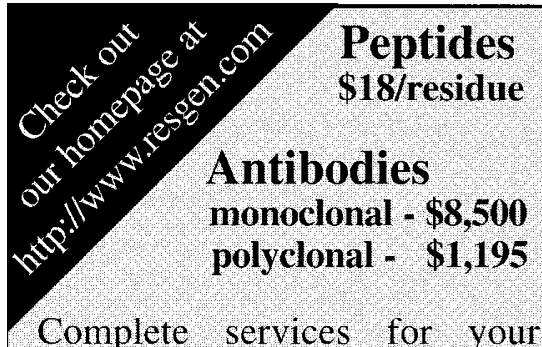

Complete services for your custom peptide and polyclonal or monoclonal antibody needs.

Research Genetics, Inc.

U.S. or Canada 800-533-4363

U.K. 0-800-89-1393

FAX 256-536-9016

READER ENQUIRY NO.37 
nucleic acid sequencing, nucleic acid probe preparation and purification, the isolation and enrichment of cDNAs, single-strand DNA labelling and library construction, and specific hybridization. MPG LCA (longchain alkylamine) is used for ligand attachment. It is derivitized to provide amino groups that offer greater flexibility for binding a wide variety of ligands. The long, $15 \AA$ spacer arm present on the surface of the particle enhances reactivity by minimizing steric hindrance. MPG LCA beads can be used for the purification of nucleic acids, cDNA amplifications and subtractive hybridization. Reader Enquiry No. 112

\section{Making things gel}

\section{AlphaArchiver 600}

From Alpha Innotech

Designed as an entry-level, fully upgradeable, gel documentation system

According to the manufacturer, this system provides documentation and archiving of gels, films and membranes in just minutes. It utilizes CCD-camera technology and optics to capture real-time (30-fps) images, at $762 \times 494(\mathrm{~V} \times \mathrm{H})$ pixel resolution and greater than $56 \mathrm{db}$ signal-to-noise ratio. It can also perform low-light, chemiluminescence applications with the addition of the DE-300 EconoImage light cabinet or the DE-400 MultiImage light cabinet. It is also available with several printing options for hard copy results in seconds rather than minutes. A fully integrated software package is also provided for image documentation and archiving in most current file formats.

Reader Enquiry No. 113

\section{EZ Load molecular rulers}

From Bio-Rad

Precision-sized DNA markers, blended with loading buffer for ease and convenience

These rulers may be loaded directly onto the electrophoresis gel. They are available in six size ranges: $20-1,000 \mathrm{bp}, 10-1,000 \mathrm{bp}$, $100-3,000 \mathrm{bp}, 500-8,000 \mathrm{bp}$ and $1-15 \mathrm{~kb}$, as well as a precision molecular mass standard. According to the manufacturer, there is no spurious migration from sequence variation and no contaminating plasmid DNA. Visually distinct reference bands simplify readability, and the rulers progress by precise, easily calculable increments for easy size determination, says Bio-Rad.

Reader Enquiry No. 114

\section{DNA ladders}

From Flowgen

\section{Four DNA ladders for DNA electrophoresis}

These new ladders now cover molecular sizes from $20 \mathrm{bp}$ to $8 \mathrm{~kb}$ in steps of 20,100 or $500 \mathrm{bp}$, and are said to be free from contaminating plasmid or 'junk' DNA. The first of the four available ladders is a 20-bp increment DNA ladder, consisting of 50 fragments in the range from 20 to $1,000 \mathrm{bp}$. The 100-bp ladder consists of ten fragments from $100-1,000 \mathrm{bp}$ in 100-bp increments. There is also a 100-bp, extended-range ladder with over 30 fragments covering the range of $100 \mathrm{bp}$ to $3,000 \mathrm{bp}$ in 100-bp increments. The last member of the group is a 500 -bp version with 16 fragments from 500 bp to $8 \mathrm{~kb}$, occurring at 500-bp increments. Reader Enquiry No. 115

\section{astbutnotleast}

S120H oven/shaker

From Merck

\section{A hybridization oven/shaker from}

Stuart Scientific

This unit provides a value alternative for those undertaking hybridizations or general shaking/incubator procedures. Supplied by Merck throughout Europe, the S120H features high-performance temperature control, using forced air circulation and a variable-speed, seven-bottle rotisserie. It has been designed for the widely used $20-\mathrm{cm}$ wide hybridization membrane. The door is counterbalanced to allow for easy opening and designed to ensure full front and top access. The rotisserie is easily removed and serves also as a bottle stand.

Reader Enquiry No. 116

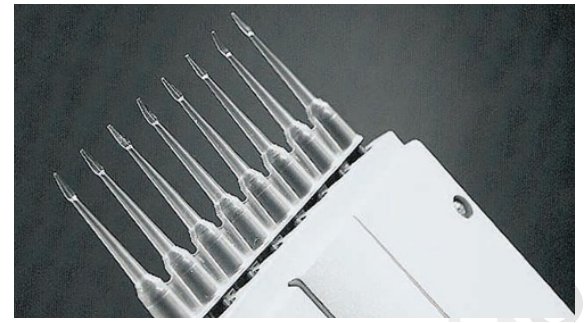

Hot tips — SequaStrip tips from Embi Tec.

\section{SequaStrip tips}

From Embi Tec

A system that is touted to allow the loading of sequencing gels in as little as five minutes

According to the manufacturer, this is the only eight-tip strip and multichannel pipette combination designed specifically for DNA sequencing. The tips are short with a very flat, narrow paddle and the strip is straight, which is said to result in a simple loading action. For a precise draw each time, the pipette is calibrated. The strips were designed for sequencing systems and fit $0.2-, 0.3-$ and $0.4-\mathrm{mm}$ gels. The tips are disposable, to help eliminate broken glass or cross-contamination.

Reader Enquiry No. 117

These notes are compiled by Brendan Horton

from information provided by the manufacturers. For more details, fill in the reader service card bound inside the journal.

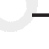

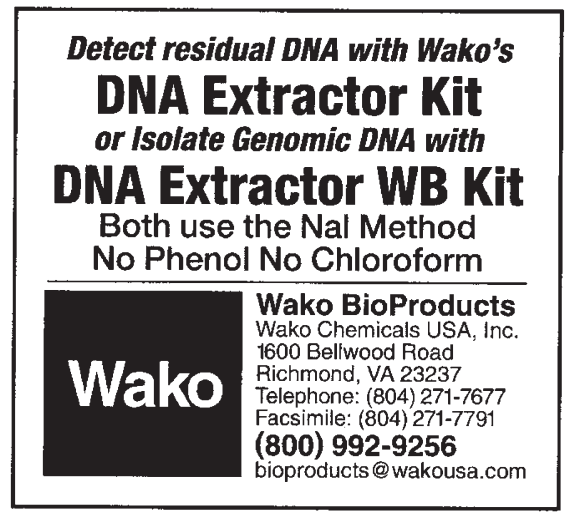

READER ENQUIRY NO.35

\section{Greenland Summit Ice Cores CD-ROM}

Cores from the Greentand Summit provide information on environmental change. Data measurements include major ions, isotopes, sulfate, nitrate, $\mathrm{CO} 2$, ecm, etc To order a CD-ROM contact: nsidc@kryos.colorado.edu Phone +1 (303) 4926199 Fax +1 (303) 4922468

Data are available electronically at: http://arcss.colorado.edu

\section{NeW Severn Super Sequencer \\ Acrylamide Gel Solution}

Severn Super Sequencer is a new improved gel solution for use in automated DNA sequencing. Complete with denaturant and buffer, it comes in a series of 'ready to use $^{\prime}$ (RTU) formulations. Simply pour and Polymerise.

So in the race to sequence, don't walk, RUN!

With Severn Super Sequencer - Available in $100 \mathrm{ml}, 500 \mathrm{ml}$ and $5000 \mathrm{ml}$ volumes.

\section{Trial $100 \mathrm{ml}$ available on request.}

\section{Severn Biotech Ltd}

Severn Biotech Ltd, Unit 2, Park Lane, Stourport Road, Kidderminster, Worcs DY11 6TJ, U.K. Tel: (01562) 825286 Fax: (01562) 825284 E.Mail: 101626.2102@ Compuserve.com UK Manufacturers of Liquid Acrylamides, Buffers and Electrophoretic Media READER ENQUIRY NO.25 